\title{
Robust Fault Detection for Permanent-Magnet Synchronous Motor via Adaptive Sliding-Mode Observer
}

\author{
Miaoying Zhang $\mathbb{D},{ }^{1}$ Fan Xiao $\mathbb{D}^{2},{ }^{2}$ Rui Shao $\mathbb{D}^{1},{ }^{1}$ and Zhaojun Deng $\mathbb{D}^{3}$ \\ ${ }^{1}$ College of Railway Transportation Locomotive and Vehicle College, Hunan Railway Professional Technology College, Zhuzhou, \\ Hunan, China \\ ${ }^{2}$ College of Electrical and Information Engineering, Hunan University of Technology, Zhuzhou, Hunan, China \\ ${ }^{3}$ College of Railway Transportation Operation and Management, Hunan Railway Professional Technology College, Zhuzhou, \\ Hunan, China
}

Correspondence should be addressed to Fan Xiao; 511191117@qq.com

Received 8 February 2020; Accepted 10 April 2020; Published 30 April 2020

Guest Editor: Hou-Sheng Su

Copyright (c) 2020 Miaoying Zhang et al. This is an open access article distributed under the Creative Commons Attribution License, which permits unrestricted use, distribution, and reproduction in any medium, provided the original work is properly cited.

In this paper, a robust fault detection problem is investigated for a permanent magnet synchronous motor (PMSM). By using the adaptive control and the sliding-mode control strategies, an observer is presented for estimating the amplitude demagnetization fault problem under inductance disturbance. The proposed method can effectively attenuate the inductance disturbance of the PMSM by the sliding-mode strategy. And the adaptive control estimation algorithm is adopted for guaranteeing that the real-time detection of demagnetization flux can be realized. The convergency is obtained by the Lyapunov stability theory. Finally, simulation is given for demonstrating the feasibility and effectiveness of the proposed method.

\section{Introduction}

With its high efficiency, low energy consumption, lightweight, low noise, maintainability, and many other advantages [1-3], the permanent-magnet synchronous motor (PMSM) has absorbed strong interest from many scholars. For the rapid development of rail transit, new energy vehicles, aviation, ships, and other fields, the application of the PMSM is becoming more and more widespread, and its research has become a hotspot of traction power.

In recent years, the study on stability of the PMSM has been highly concerned by researchers, and some achievements have been reported $[4,5]$. In addition to the influence of various disturbance factors, the demagnetization of permanent magnet caused by disturbance is also a great threat to the stability of the PMSM. Due to the special permanent-magnet structure of the PMSM, the magnetization performance will decline or demagnetize under the disturbance. Once the permanent magnet demagnetizes, the PMSM fails to run stably and it will be even scrapped [6].
Therefore, the demagnetization detection of the PMSM is particularly important. The sliding-mode control is of great theoretical research significance on the stability of the PMSM because it is insensitive to model error and external disturbance of the research object [7-9]. The adaptive control can modify the control process according to the change of the dynamic characteristics of the controlled object and the disturbance. Its research object can contain the objective disturbance, and the adaptive control estimation algorithm can make a specified performance index reach and maintain the optimal state or approximately optimal state [10]. The sliding-mode control and the adaptive control with their unique advantages have made great improvement in recent years. Many scholars have conducted research studies on them and obtained a large number of excellent results [11-25].

At present, the sliding-mode control and the adaptive control in the PMSM are mainly used in speed control [11, 12], rotor position estimation [13], parameter identification [14], and other research fields. As for the sliding-mode 
control, [15] introduces a hybrid controller (HC) for the speed control of the PMSM drive. Hosseyni et al. [16] present a speed control technique based on the sliding-mode observer (SMO) and the backstepping controller to estimate rotor speed and rotor position. The literature [14] implements parameter identification under parameter disturbance with the sliding-mode control. For the adaptive control, [17] proposes an adaptive PID controller for measurement and control of position or speed. Nguyen et al. [18] propose a simple adaptive control-based speed controller under parameter disturbance. Qutubuddin and Yadaiah [19] present an intelligent adaptive mechanism to estimate rotor speed, rotor position, and motor parameter identification.

In addition to separate the sliding-mode control and the adaptive control, there are also related studies that combine the two control strategies. Liu et al. [20] propose an adaptive sliding-mode control observer under the parameter disturbance. Xi et al. [12] propose a speed SMC with an adaptive law and an extended state observer (ESO).

In the above literature, the application of the slidingmode control and the adaptive control in the PMSM has involved disturbance, but not including the flux demagnetization of the PMSM. In the actual operation process, disturbance will often cause demagnetization, and if demagnetization is not discovered and handled in time, the PMSM will be scrapped if demagnetization goes serious. Therefore, many scholars have studied the flux-linkage detection of the PMSM [21-24]. Zhang et.al. [22] address permanent-magnet demagnetization fault in the PMSM, but no disturbance is involved. In [23], a stator flux-linkage observer based on the phase-locked loop is introduced which aims at the low-accuracy stator flux-linkage estimation in traditional direct control. In [24], a minimum-order extended flux-linkage sliding-mode observer is presented which can accurately observe torque and permanent-magnet flux.

These research studies on the permanent-magnet demagnetization fault detection of the PMSM do not consider the parameter change or only consider the resistance parameter change, so this paper selects a new field based on all above studies and proposes an observer which combines the slidingmode control with the adaptive control aiming at the condition of the inductance disturbance. The characteristics of the sliding-mode control strategy are not sensitive to parameter and can overcome the inductance disturbance of the PMSM. The real-time adaptive control estimation algorithm is presented for realizing the real-time amplitude demagnetization fault detection of permanent-magnet flux linkage.

In this paper, the PMSM is taken as the main research object, and its stable control theory and real-time amplitude demagnetization fault detection of flux linkage are studied. Its successful development will not only enrich the research studies on the demagnetization fault detection theory of the PMSM but also be applied to further research studies about more disturbances for the PMSM on this basis.

\section{Description of the Problem}

In magnetic field-oriented coordinate $d-q$, the mathematical model [25] of demagnetization current of the PMSM is

$$
\left\{\begin{array}{l}
\frac{\mathrm{d} i_{d}}{\mathrm{~d} t}=\frac{u_{d}}{L}-\frac{R}{L} i_{d}+\omega i_{q}+\omega \frac{\psi_{r q}}{L} \\
\frac{\mathrm{d} i_{q}}{\mathrm{~d} t}=\frac{u_{q}}{L}-\frac{R}{L} i_{q}-\omega i_{d}-\omega \frac{\psi_{r d}}{L}
\end{array}\right.
$$

where $i_{d}$ and $i_{q}$ are the current of the $d-q$ axis, $u_{d}$ and $u_{q}$ are the voltage of the $d-q$ axis, $R$ is the resistance, $\omega$ is the angular velocity, $L$ is the inductance, $\psi_{r d}$ is the flux linkage of the $d$ axis, and $\psi_{r q}$ is the flux linkage of $q$ axis.

The permanent-magnet flux-linkage change of the PMSM is shown in Figure 1. $A B C$ is the three-phase static coordinate, and axis $A$ is coincidence with the axis of the actual stator $A$ phase winding. $d-q$ is the magnetic fieldoriented coordinate, and $d$ axis points to the direction of rotor permanent-magnet flux-linkage and is coincidence with the magnetic pole axis of the rotor. $\psi_{f}$ is flux-linkage under normal circumstances, and $\theta$ is the intersection angle between $\psi_{f}$ and the axis of $A$ phase stator winding of motor. $\psi_{f}$ rotates at angular velocity $\omega$ in space along with the rotor, and $q$ axis is $90^{\circ}$ ahead of $d$ axis counterclockwise. Deviation angle between direction of magnetic field orientation and direction of permanent-magnet flux-linkage direction is $\gamma$.

For amplitude demagnetization, demagnetization direction of the permanent-magnet flux linkage always occurs in the same direction of $d$ axis, which has no effect on $q$ axis. Formula (1) can be simplified as

$$
\left\{\begin{array}{l}
\frac{\mathrm{d} i_{d}}{\mathrm{~d} t}=\frac{u_{d}}{L}-\frac{R}{L} i_{d}+\omega i_{q} \\
\frac{\mathrm{d} i_{q}}{\mathrm{~d} t}=\frac{u_{q}}{L}-\frac{R}{L} i_{q}-\omega i_{d}-\omega \frac{\psi_{r d}}{L}
\end{array}\right.
$$

Under the inductance disturbance, the demagnetization mathematical model is given as follows:

$$
\left\{\begin{array}{l}
\frac{\mathrm{d} i_{d}}{\mathrm{~d} t}=\frac{u_{d}}{L+\Delta L}-\frac{R}{L+\Delta L} i_{d}+\omega i_{q} \\
\frac{\mathrm{d} i_{q}}{\mathrm{~d} t}=\frac{u_{q}}{L+\Delta L}-\frac{R}{L+\Delta L} i_{q}-\omega i_{d}-\omega \frac{\psi_{r d}}{L+\Delta L}
\end{array}\right.
$$

where $\Delta L$ is the inductance disturbance.

We let $1 /(L+\Delta L)=(1 / L)-(1 / m)$, where $m=\left(L^{2} / \Delta L\right)+L$, and equation (3) transfers into

$$
\left\{\begin{array}{l}
\frac{\mathrm{d} i_{d}}{\mathrm{~d} t}=\frac{u_{d}}{L}-\frac{R}{L} i_{d}+\omega i_{q}-\frac{u_{d}}{m}+\frac{R}{m} i_{d}, \\
\frac{\mathrm{d} i_{q}}{\mathrm{~d} t}=\frac{u_{q}}{L}-\frac{R}{L} i_{q}-\omega i_{d}-\omega \frac{\psi_{r d}}{L}-\frac{u_{q}}{m}+\frac{R}{m} i_{q}+\omega \frac{\psi_{r d}}{m} .
\end{array}\right.
$$

The state equation of the PMSM is obtained from equation (4): 


$$
\left\{\begin{array}{l}
\dot{x}=A x+B u+E f+d \\
y=C x
\end{array}\right.
$$

where state variables are $\left\{\begin{array}{l}x_{1}=i_{d} \\ x_{2}=i_{q}\end{array}, x=\left[\begin{array}{l}x_{1} \\ x_{2}\end{array}\right], u=\left[\begin{array}{l}u_{d} \\ u_{q}\end{array}\right]\right.$, $f=\left[\begin{array}{c}0 \\ \psi_{r d}\end{array}\right]$, and

$$
d=\left[\begin{array}{c}
-\frac{u_{d}}{m}+\frac{R}{m} i_{d} \\
u_{q}+\frac{R}{m} i_{q}+\omega \frac{\psi_{r d}}{m}
\end{array}\right],
$$

and coefficient matrixes are

$$
\begin{aligned}
& A=\left[\begin{array}{cc}
-\frac{R}{L} & \omega \\
-\omega & -\frac{R}{L}
\end{array}\right], \\
& B=\left[\begin{array}{ll}
\frac{1}{L} & 0 \\
0 & \frac{1}{L}
\end{array}\right], \\
& E=-\frac{\omega}{L}, \\
& C=\left[\begin{array}{ll}
1 & 0 \\
0 & 1
\end{array}\right] .
\end{aligned}
$$

\section{Design of Adaptive Sliding-Mode Observer}

For the PMSM system described in (5), an adaptive slidingmode observer is constructed as follows:

$$
\left\{\begin{array}{l}
\dot{\hat{x}}=A \hat{x}+B u+k \operatorname{sgn}(x-\hat{x})+E \hat{f}+G(y-\hat{y}), \\
\hat{y}=C \hat{x}
\end{array}\right.
$$

where $\operatorname{sgn}(x-\hat{x})$ is the symbolic function, $k$ is the to-bedesigned constant, $G$ is the to-be-designed matrix, superscript " $\wedge$ " is the observed value of relevant disturbances, $k \operatorname{sgn}(x-\hat{x})$ is used to counteract influence of the parameter disturbance, $\hat{f}$ is used to observe the demagnetization of the permanent-magnet flux linkage, and $G(y-\widehat{y})$ is used to control the convergence rate and optimize the stability of the system state.

Observer deviation is defined as $e=x-\hat{x}=$ $\left[\begin{array}{l}e_{1} \\ e_{2}\end{array}\right]=\left[\begin{array}{l}x_{1}-\hat{x}_{1} \\ x_{2}-\widehat{x}_{2}\end{array}\right]$

Output deviation is defined as $e_{y}=y-\hat{y}$.

From formulas (5) and (8), we obtain

$$
\begin{aligned}
\dot{e} & =\dot{x}-\dot{\hat{x}} \\
& =(A-G C) e+E e_{f}+d-k \operatorname{sgn}(x-\hat{x}),
\end{aligned}
$$

where fault estimation error $e_{f}=f-\widehat{f}$.

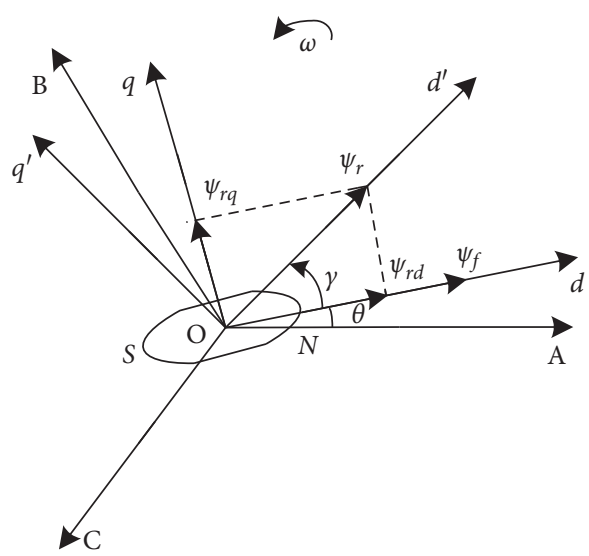

Figure 1: Permanent-magnet flux-linkage change of the PMSM.

The improved adaptive control estimation algorithm proposed in this paper is

$$
\dot{\widehat{f}}=-\Gamma F\left(\dot{e}_{y}+e_{y}\right),
$$

where $\Gamma=\Gamma^{T}>0$ is the adaptive learning rate and $F$ is an optimal dimensional matrix which is the to-be-designed observer gain matrix.

Remark 1. Based on the improved adaptive control estimation algorithm (10), we can obtain the following real-time permanent-magnet flux-linkage estimation value:

$$
\widehat{f}=-\Gamma \int F\left(\dot{e}_{y}+e_{y}\right) .
$$

Lemma 1. For any positive number $\mu$ and symmetric positive definite matrix $P$, the following inequality holds:

$$
2 x^{T} P y \leq \frac{1}{\mu} x^{T} P x+\mu y^{T} P y .
$$

Theorem 1. For formula (8), if $k$ is large enough, a symmetric positive definite matrix $F \in R^{r \times p}$ satisfies

$$
\Theta=\left[\begin{array}{cc}
2(A-G C) & F C(A-G C)+F C+E \\
* & 2 F C E+H
\end{array}\right]<0 .
$$

Improved adaptive control estimation algorithm such as formula (11) makes the state estimation error and the fluxlinkage estimation error converge to zero.

\section{Convergency Analysis}

Choosing the following positive definite function as Lyapunov function, we can get

$$
V=e^{T} e+e_{f}^{T} \Gamma^{-1} e_{f}
$$

The derivative of formula (14) is 


$$
\begin{aligned}
\dot{V}= & 2 e^{T} \dot{e}+2 e_{f}^{T} \Gamma^{-1} \dot{e}_{f} \\
= & 2 e^{T}(A-G C) e+2 e^{T} E e_{f}+2 e^{T}(d-k \operatorname{sgn}(x-\hat{x})) \\
& +2 e_{f}^{T} \Gamma^{-1}(f-\dot{\hat{f}}) \\
= & 2 e^{T}(A-G C) e+2 e^{T} E e_{f}+2 e^{T}(d-k \operatorname{sgn}(x-\widehat{x})) \\
& +2 e_{f}^{T} \Gamma^{-1} \dot{f} \\
& +2 e_{f}^{T}(F C(A-G C)+F C) e+2 e_{f}^{T} F C E e_{f} \\
& +2 e_{f}^{T} F C(d-k \operatorname{sgn}(x-\hat{x})) .
\end{aligned}
$$

Let

$$
\dot{V}=V_{1}+V_{2},
$$

formula (16) can be divided into

$$
\begin{aligned}
V_{1}= & 2 e^{T}(d-k \operatorname{sgn}(x-\widehat{x}))+2 e_{f}^{T} F C(d-k \operatorname{sgn}(x-\widehat{x})), \\
V_{2}= & 2 e^{T}(A-G C) e+2 e_{f}^{T}(F C(A-G C)+F C) e \\
& +2 e_{f}^{T} F C E e_{f}+2 e^{T} E e_{f}+2 e_{f}^{T} \Gamma^{-1} f .
\end{aligned}
$$

(a) For $V_{1}$,

$$
\begin{array}{r}
V_{1}=2 e^{T}(d-k \operatorname{sgn}(x-\hat{x}))+2 e_{f}^{T} F C(d-k \operatorname{sgn}(x-\hat{x})) \\
\leq 2\|e\|\|d\|-2 k\|e\|+2\left\|e_{f}\right\|\|F C\|(\|d\|-k) \\
=2\|e\|(\|d\|-k)+2\left\|e_{f}\right\|\|F C\|(\|d\|-k) .
\end{array}
$$

From the engineering point of view, both the stator resistance and the permanent-magnet flux- linkage are bounded. Thus, it can be assumed that $d$ is bounded, that is, there is a positive constant $\|d\| \leq \varepsilon$, which makes $\|d\| \leq \varepsilon$ bounded (the mathematical symbol represents the Euclidean norm of vector or spectral norm of matrix)

If $k$ is large enough, it satisfies

$$
\|d\| \leq \varepsilon<k \text {. }
$$

From formula (18), the following formula is obtained:

$2 e^{T}\left(d-k \operatorname{sgn}(x-\widehat{x})+2 e^{T} E e_{f}-2 e_{f}^{T} F C k \operatorname{sgn}(x-\hat{x})\right)<0$.

(b) For $V_{2}$,

$$
\begin{aligned}
V_{2}= & 2 e^{T}(A-G C) e+2 e_{f}^{T}(F C(A-G C)+F C) e \\
& +2 e_{f}^{T} F C E e_{f}+2 e^{T} E e_{f}+2 e_{f}^{T} \Gamma^{-1} f .
\end{aligned}
$$

TABLE 1: The setting value of motor parameters.

\begin{tabular}{lc}
\hline Motor parameters $\Omega$ & The setting value \\
\hline Resistance & 2.875 \\
Number of pole-pairs & 4 \\
Inductance (H) & 0.0085 \\
The rotor flux linkage (Wb) & 0.175 \\
The rotor inertia (Kgm) & 0.008 \\
\hline
\end{tabular}

According to Lemma 1, we obtain

$$
\begin{array}{r}
2 e_{f}^{T} \Gamma^{-1} f \leq e_{f}^{T} H e_{f}+f^{T} \Gamma^{-1} H \Gamma^{-1} f \\
\quad \leq e_{f}^{T} H e_{f}+f^{2} \lambda_{\max }\left(\Gamma^{-1} H \Gamma^{-1}\right),
\end{array}
$$

where $H$ is a symmetric positive definite matrix.

We can obtain

$$
\begin{aligned}
\dot{V}_{2} \leq & 2 e^{T}(A-G C) e+2 e_{f}^{T}(F C(A-G C)+F C) e \\
& +2 e_{f}^{T} F C E e_{f}+2 e^{T} E e_{f} \\
& +e_{f}^{T} H e_{f}+f^{2} \lambda_{\max }\left(\Gamma^{-1} H \Gamma^{-1}\right) \\
= & Z^{T} \Theta Z+\delta .
\end{aligned}
$$

Here, $Z=\left[\begin{array}{c}e \\ e_{f}\end{array}\right], \Theta=\left[\begin{array}{cc}2(A-G C) F C(A-G C)+F C+E \\ * & 2 F C E+H\end{array}\right]$, and $\delta=f^{2} \lambda_{\max }\left(\Gamma^{-1} H \Gamma^{-1}\right)$.

If formula (13) holds, let $\sigma=\lambda_{(\min )}(-\Theta)$.

We obtain

$$
\begin{aligned}
\dot{V} \leq & -\sigma\|Z\|^{2}+\delta+2 e^{T}(d-k \operatorname{sgn}(x-\hat{x})) \\
& +2 e_{f}^{T} F C(d-k \operatorname{sgn}(x-\hat{x})) \leq 0 .
\end{aligned}
$$

When $\|Z\|^{2} \geq(\delta / \sigma), k$ and $F$ are large enough, $\dot{V} \leq 0$, that is, $\left\langle Z \mid\|Z\|^{2} \geq(\delta / \sigma)\right\rangle$, which makes the state estimation error $e$ and the permanent-magnet flux-linkage estimation error $e_{f}$ converge to zero.

\section{Simulation Analysis}

The parameters of the PMSM used in simulation are shown in Table 1. Meanwhile, the initial value of the observer is set to 0 .

The load torque disturbance changes from $0 \mathrm{Nm}$ to $20 \mathrm{Nm}$ at $0.05 \mathrm{~s}$. The inductance disturbance changes from $0.0085 \mathrm{H}$ to $0.0105 \mathrm{H}$ at $0.3 \mathrm{~s}$. Amplitude demagnetization changes from $0.175 \mathrm{~Wb}$ to $0.17 \mathrm{~Wb}$ at $0.4 \mathrm{~s}$.

The parameters of the observer module are as follows:

$$
\begin{aligned}
G & =\left[\begin{array}{cc}
10 & 0 \\
0 & 100
\end{array}\right], \\
k & =\left[\begin{array}{cc}
40000 & 0 \\
0 & 1000
\end{array}\right], \\
\Gamma & =\left[\begin{array}{cc}
67 & \\
& 180
\end{array}\right] .
\end{aligned}
$$




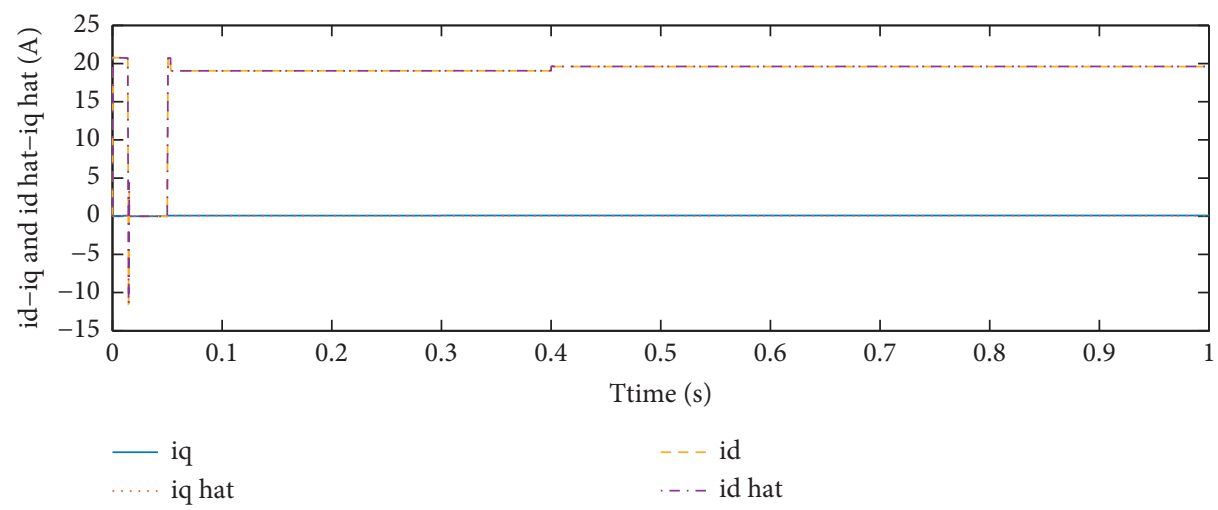

FIgURE 2: Simulation waveforms of $i_{d}, \hat{i}_{d}, i_{q}$, and $\widehat{i}_{q}$.

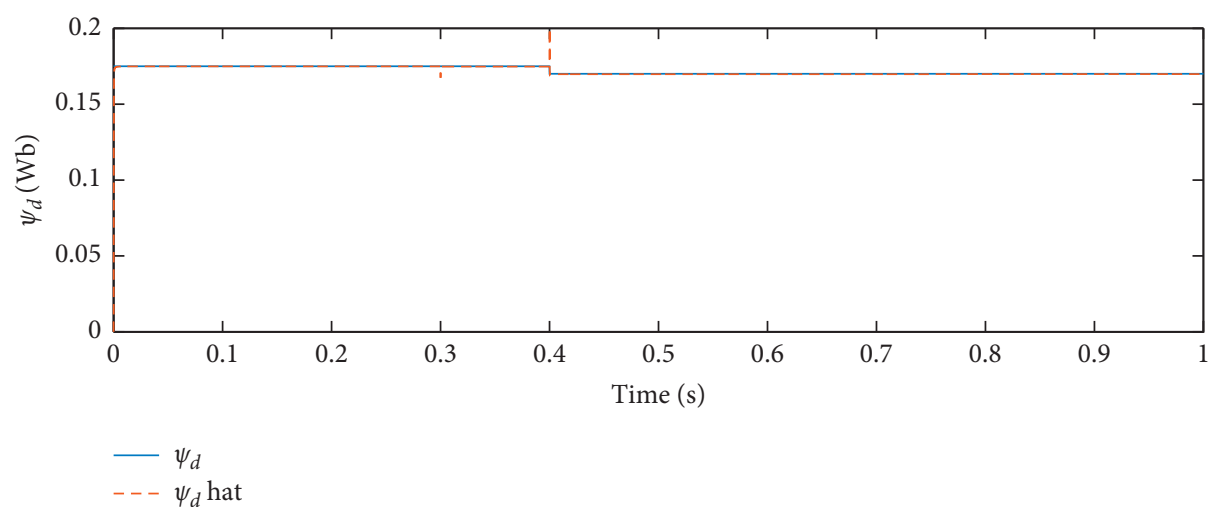

FIgURE 3: Simulation waveforms of $\psi_{r d}$ and $\widehat{\psi}_{r d}$.

As is shown in Figure 2, when the PMSM is loaded at $0.05 \mathrm{~s}$, the current $i_{q}$ jumps from the initial value $0 \mathrm{~A}$ to $19 \mathrm{~A}$. But when the inductance changes at $0.3 \mathrm{~s}, i_{q}$ is almost not affected. When an amplitude demagnetization fault occurs at $0.4 \mathrm{~s}, i_{q}$ increases slightly. We can conclude that only the loading makes $i_{q}$ increase by a large margin, the demagnetization fault has little effect on $i_{q}$, and the inductance parameter variation of the PMSM basically has nothing to do with $i_{q}$. We use $i_{d}=0$ control algorithm, so the current $i_{d}$ always remains at $0 \mathrm{~A} . i_{d}$ shakes only when the load torque disturbance occurs, and then it quickly returns to $0 \mathrm{~A}$.

As is shown in Figure 3, $\widehat{\psi}_{r d}$ is not affected when the PMSM is loaded at $0.05 \mathrm{~s}$ and it shakes when the inductance disturbance occurs at $0.3 \mathrm{~s}$. When an amplitude demagnetization occurs at $0.4 \mathrm{~s}, \psi_{r d}$ decreases from $0.175 \mathrm{~Wb}$ to $0.170 \mathrm{~Wb}$ and $\widehat{\psi}_{r d}$ shakes and then quickly keeps up with $\psi_{r d}$.

Matlab simulation results verify the feasibility and effectiveness of the theoretical method.

\section{Conclusion}

During the operation of the PMSM, it is affected by disturbances and demagnetization fault. The inductance is one of the important disturbances for the PMSM, so an adaptive sliding-mode observer is proposed to realize the amplitude demagnetization fault robust detection method with inductance disturbance for the PMSM in this paper.
Firstly, a mathematical model of the demagnetization fault of the PMSM in the d-q coordinate is established. Then, an adaptive control estimation algorithm is designed to realize the amplitude demagnetization fault detection of the permanent-magnet flux-linkage, while the sliding-mode control strategy overcomes the inductance disturbance. Finally, the Matlab simulation results show the effectiveness of the adaptive sliding-mode observer.

\section{Data Availability}

There do not exist supporting data regarding this manuscript.

\section{Conflicts of Interest}

The authors declare that they have no conflicts of interest.

\section{References}

[1] G. H. B. Foo, X. Zhang, and D. M. Vilathgamuwa, "A sensor fault detection and isolation method in interior permanentmagnet synchronous motor drives based on an extended Kalman filter," IEEE Transactions on Industrial Electronics, vol. 60 , no. 8, pp. 3485-3495, 2013.

[2] S. Chakraborty, E. Keller, A. Ray, and J. Mayer, "Detection and estimation of demagnetization faults in permanent magnet synchronous motors," Electric Power Systems Research, vol. 96, pp. 225-236, 2013. 
[3] J. Mao, Unifying Electrical Engineering and Electronics Engineering, Springer, Berlin, Germany, 2013.

[4] W. Zhao, A. Yang, J. Ji, Q. Chen, and J. Zhu, "Modified flux linkage observer for sensorless direct thrust force control of linear vernier permanent magnet motor," IEEE Transactions on Power Electronics, vol. 34, no. 8, pp. 7800-7811, 2019.

[5] H. Su, J. Zhang, and X. Chen, "A stochastic sampling mechanism for time-varying formation of multiagent systems with multiple leaders and communication delays," IEEE Transactions on Neural Networks and Learning Systems, vol. 30, no. 12, pp. 3699-3707, 2019.

[6] C. Zhang, M. Zhang, J. He et al., "Demagnetization faults robust detection method based on an adaptive sliding mode observer for PMSM," Journal of Advanced Computational Intelligence and Intelligent Informatics, vol. 20, no. 7, pp. 1127-1134, 2016.

[7] Y. Shu and Z. Kong, "Adaptive sliding mode variable structure control for a new hyper chaos system," Journal of Chongqing University of Technology (Natural Science), vol. 24, no. 11, pp. 109-112, 2010, in Chinese.

[8] C. Xu, H. Su, C. Liu, and G. Zhang, "Robust adaptive synchronization of complex network with bounded disturbances," Advances in Difference Equations, vol. 2019, no. 1, p. 483, 2019.

[9] C. Xu, Y. Zhao, B. Qin, and H. Zhang, "Adaptive synchronization of coupled harmonic oscillators under switching topology," Journal of the Franklin Institute, vol. 356, no. 2, pp. 1067-1087, 2019.

[10] R. Kandiban and R. Arulmozhiyal, "Design of adaptive fuzzy PID controller for speed control of BLDC motor," International Journal of Soft Computing and Engineering, vol. 2, no. 1, pp. 386-391, 2012.

[11] M. A. M. Cheema, J. E. Fletcher, M. Farshadnia, D. Xiao, and M. F. Rahman, "Combined speed and direct thrust force control of linear permanent-magnet synchronous motors with sensorless speed estimation using a sliding-mode control with integral action," IEEE Transactions on Industrial Electronics, vol. 64, no. 5, pp. 3489-3501, 2017.

[12] P. Xi, Y. Deng, Z. Wang, and H. Li, "Speed adaptive sliding mode control with an extended state observer for permanent magnet synchronous motor," Mathematical Problems in Engineering, vol. 2018, Article ID 6405923, 13 pages, 2018.

[13] B. Du, S. Han, C. Zhang et al., "Sensorless control of interior permanent magnet synchronous motor based on active disturbance rejection controller," Transactions of China Electrotechnical Society, vol. 32, no. 3, pp. 105-112, 2017, in Chinese.

[14] X. Zhang and Z. Li, "Sliding-mode observer-based mechanical parameter estimation for permanent magnet synchronous motor," IEEE Transactions on Power Electronics, vol. 31, no. 8, pp. 5732-5745, 2016.

[15] C. Elmas and O. Ustun, "A hybrid controller for the speed control of a permanent magnet synchronous motor drive," Control Engineering Practice, vol. 16, no. 3, pp. 260-270, 2008.

[16] A. Hosseyni, R. Trabelsi, M. Mimouni et al., "Sensorless backstepping control for a five-phase permanent magnet synchronous motor drive based on sliding mode observer," Lecture Notes in Electrical Engineering, pp. 251-261, Springer, Berlin, Germany, 2016.

[17] M. Geethu and P. Kunjumon, "Sensorless adaptive PID speed control for permanent magnet synchronous motor drives," in Proceedings of the 2016 International Conference on Emerging Technological Trends, IEEE, Kollam, India, October 2016.
[18] A. T. Nguyen, M. S. Rafaq, H. H. Choi, and J.-W. Jung, “A model reference adaptive control based speed controller for a surface-mounted permanent magnet synchronous motor drive," IEEE Transactions on Industrial Electronics, vol. 65, no. 12, pp. 9399-9409, 2018.

[19] M. Qutubuddin and N. Yadaiah, "A new intelligent adaptive mechanism for sensorless control of permanent magnet synchronous motor drive," Biologically Inspired Cognitive Architectures, vol. 24, pp. 47-58, 2018.

[20] J. Liu, H. Li, and Y. Deng, "Current adaptive sliding mode control based on disturbance observer for permanent magnet synchronous motor," Optics and Precision Engineering, vol. 25, no. 5, pp. 1229-1241, 2017.

[21] W. Xu, Y. Jiang, C. Mu, and F. Blaabjerg, "Improved nonlinear flux observer-based second-order SOIFO for PMSM sensorless control," IEEE Transactions on Power Electronics, vol. 34, no. 1, pp. 565-579, 2019.

[22] C. Zhang, G. Wu, J. He, and K. Zhao, "Sliding observer-based demagnetisation fault-tolerant control in permanent magnet synchronous motors," The Journal of Engineering, vol. 2017, no. 6, pp. 175-183, 2017.

[23] H. Zhu and Z. Zhao, "Direct control of bearingless permanent magnet slice motor based on novel flux linkage observer," Proceedings of the CSEE, vol. 38, no. 14, pp. 4242-4251, 2018.

[24] G. Huang, Y. Luo, C. Zhang, and K. Zhao, "Online demagnetization detection of permanent magnet synchronous traction motor based on extended flux linkage," Journal of the China Railway Society, vol. 38, no. 2, pp. 48-55, 2016, in Chinese.

[25] H. Jin and J. Huang, "Adaptive flux estimation and parameters identification of induction motors based on model reference approach," Transactions of China Electrotechnical Society, vol. 21 , no. 1, pp. 65-69, 2006, in Chinese. 